\title{
Studien über die Eiweisskörper sowie den kolloid=osmotischen Druck des den Skelettmuskel durchspïlenden Blutes.
}

2. Mitteilung: Versuch an Hunden mit dem Nierenfunktionsausfall.

$$
\text { Von }
$$

Sigetaka Kusano.

(草野重孝)

(Aus der Medizinischen Klinik von Prof. Dr. T. Kato, T'ohoku Reichsuniversität zu Sendai.)

In der ersten Mitteilung ${ }^{1)}$ meiner einschlägigen Studien ist experimentell erwiesen worden, dass das Muskelgewebe, welches über den grössten Teil der Eiweisskörper im Organismus verfügt, zum Verhalten des Eiweisses des Blutes und dessen kolloid-osmotischen Drucks (k.o. D.) in innigsten Beziehung steht, indem es nötigenfalls seine Depoteiweisskörper ins Blut abgibt oder aus dem Blut Eiweiss in sich aufnimmt.

Dass bei Nierenkranken sowie bei experimentellen Nierenschädigungen die Eiweisskörper des Blutes quantitativ und qualitativ bedeutende Umwälzung erfahren, wobei der k.o.D. je nach den Zuständen der Erkrankungen verschieden stark absinkt, ist seit Starling ${ }^{2)}$ durch zahlreiche klinische sowie experimentelle Untersuchungen bestätigt worden (Goverts, ${ }^{3)}$ Rusznyák, ${ }^{\text {4) }}$ Iversen und Nakazawa, ${ }^{5)}$ Cope, ${ }^{6}$ ) Farkas, ${ }^{7)}$ Kylin, ${ }^{8)}$ Meyer, ${ }^{97}$ Ölkers, ${ }^{10)}$ Schenck und Schülter ${ }_{i}{ }^{11)}$

\footnotetext{
1) Ku s a n o, Tohoku Journ. Exp. Med., 1938, 34, 247.

2) Starling. Journ. of Physiol., 1899, 24, 317.

3) Goverts, Bull. Acad. Roy. Med. Belg., 1924, 4, 161.

4) Ruszn yák, Ztschr. f. d. ges. exp. Med., 1924, 41, 532.

5) Iversen u. Na kazawa, Biochem. Ztschr., 1927, 191, 307.

6) C o pe, Quart. Journ. Med., 1928, 22, 91.

7) F a r a s, Ztschr. f. d. ges. exp. Med., 1928, 62, 35.

8) Kyli n, Ibid., 1929, 68, 746.

9) Me ye r, Ztschr. f. klin. Med., 1931, I15, 647.

10) Ölkers, Tbid., 1931, 115, 854.

11) Schenck u. Schülter, Arch. f. exp. Path. u. Pharm., 1933, 169, 343.
} 
an hiesiger Klinik Kimura, K. und Nakazawa, $\left.{ }^{12}\right) \mathrm{Kimura}, \mathrm{S}$. und Nakazawa, ${ }^{13)}$ Sanada, ${ }^{14)}$ Fukuhara, ${ }^{15)} \mathrm{Hitach}^{16)}$ u.a.)

Über die Frage, worauf diese bei Nierenschädigungen zustande kommende Veränderung des Bluteiweisses zurückzuführen sei, ist bis dahin von verschiedenen Seiten studiert worden, ohne dass man dennoch zu endgültigem Resultat gekommen ist. Es haben an hiesiger Klinik Shida $\mathbf{a}^{17)}$ bei experimentellen Nierenfunktionsstörungen am die Niere zirkulierenden Blut Bestimmungen von Bluteiweiss und k. o.D., Yasuda ${ }^{18)}$ ebenfalls bei experimentellen Nierenschädigungen an dem der Leber zu- und entströmenden Blut auch einschlägige Bestimmungen vorgenommen und den Nachweis erbracht, dass sowohl die Niere wie auch die Leber bei den beim Funktionsausfall der Niere auftretenden Veränderungen des Bluteiweisses und k.o.D. eine wichtige Rolle spielt.

Es muss dann die Frage aufgeworfen werden, welches Verhalten die Muskulatur, die mit dem Schicksal des Bluteiweisses in innigem Zusammenhang steht, bei gestörter Nierenfunktion gegenüber dem Bluteiweiss sowie dem k.o.D. aufweist. Diese Frage, die im Zusammenhang mit dem Studium in meiner ersten Mitteilung ${ }^{1)}$ recht interessant und von wichtiger Bedeutung ist, steht bis dahin noch ganz offen. Von diesem Standpunkt ausgehend habe ich vorliegende Versuche angestellt, in denen ich bei der Funktionsstörung der Niere, wo bekanntermassen das Bluteiweiss Veränderungen erleiden, Eiweissanteile und k.o.D. des den Muskel zirkulierenden Blutes bestimmte, in der Hoffnung, mir ein Bild darüber zu verschaffen, wie sich der Muskel auf beide Grössen einstellt.

In vorliegenden Versuchen wurden ebenso wie in der I. Mitteilung, ${ }^{1)}$ gesunde Hunde (13-20kg Körpergewicht) verwendet, es wurden an dem dem M. gastrocnemius zuströmenden arteriellen und dem davon abströmenden venösen Blut Bestimmungen von Gesamt- $\mathrm{N}$, Rest- $\mathrm{N}$ sowie Albumin- und Globulin-N und ferner k. o. D. vorgenommen und dabei wurde eine Betrachtung darüber angestellt, welche Veränderungen oben angeführte Werte nach Durchtritt des Blutes durch den Muskel aufweisen.

12) Kimura, K. u. Nakazaw a, Tohoku Journ. Exp. Med., 1930, 16, 352,

13) Kimura, S. u. N a ka z a wa, Ibid., 1931, 18, 382.

14) S a n a d a, Ibid., 1936, 29, 156.

15) Fukuh ar a, Ibid., 1937, 30, 482 .

16) Hitati, Tohoku Igaku Zassi, 1938, 23 (bald erscheint).

17) Shid a, Nippon Naikagakkai Zassi, 1937, 25428.

18) Ya suda, Tohoku Journ. Exp. Med., 1937, 31, 456. 
Als die Methode zur Provokation der Nierenfunktionsstörungen wurde nicht der Gifte wie Uran oder Kantharidin bedient aus Rücksicht darauf, dass bei Vergiftungen mit derartigen Giften nicht nur die Niere allein, sondern auch die Leber, der Muskel und auch andere Organe primär betroffen werden. Aus dieser Erwägung heraus wurde in vorliegendem Versuch zur Methodik des Nierenfunktionsausfall die Nephrektomie und Ureterenunterbindung herangezogen. DieBestimmungsmethoden der betreffenden Daten waren ganz dieselben, die in der ersten Mitteilung ${ }^{1)}$ ausführlich geschildert wurden, weshalb ihre Beschreibung hier unterlassen werden kann.

\section{Versuch an nephrektomierten Hunden.}

Es standen 6 gut ernährte erwachsene Hunde zur Verfügung. Nachdem bei diesen Versuchstieren die beiderseitige Nephrektomie ausgeführt worden war, wurde 48-69 Stunden später der Versuch angestellt. Vor Vornahme der Nephrektomie wurden zuvörderst an dem M. gastrocnemius auf der einen Seite Bestimmungen von Eiweiss und k. o.D. im zugehörigen arteriellen und venösen Blut ausgeführt. Als durch die hernach vorgenommene Nephrektomie urämische Symptome auftraten, wurden an dem dem anderseitigen Gastrocnemius zu- und entströmenden BlutParallelbestimmungen ausgeführt, hier gewonnene Daten wurden mit zuvor erhaltenen Kontrollwerten verglichen.

Die Ergebnisse der Bestimmungen sind in Tab. 1 aufgezeichnet.

Hämoglobin erfuhr im allgemeinen, sowohl auf der arteriellen Seite wie auch auf der venösen Seite eine Verdünnung. Der Grad der Verdünnung erwies sich stets auf der venösen Seite ohne Ausnahme als geringer. Dem Durchschnittswert nach betrug die prozentische Differenz vor und nach der Ausführung der Nephrektomie auf der arteriellen Seite $-2,9$, während sie auf der venösen Seite $-1,7$ ausmachte. Es stellt sich also heraus, dass das Hämoglobin nach Durchtritt des Blutes durch den urämischen Muskel ein wenig konzentriert ist. Auch unter normalen Verhältnissen wird das Hämoglobin nach Durchtritt durch den Muskel in der Konzentration um ein Geringes erhöht. Wenn man die Grade der Hämoglobinkonzentration in beiden Blutarten vor Ausführung der Nephrektomie miteinander vergleicht, so ergibt sich, dass der Hämoglobinwert im Arterienblut im Durchschnitt 13,70 g/dl, derselbe im Venenblut 13,76 g/dl beträgt, also eine Konzentrationserhöhung von $0,4 \%$ im Venenblut erfolgt. Nach Ausführung der Nephrektomie aber wurde der Hämoglobinwert im Arterienblut zu 13,30 g/dl, 
derselbe im Venenblut zu 13,53 g/dl ermittelt, die Konzentrationserhöhung betrug also 1,7\%. Es hat sich herausgestellt, dass das Hämoglobin nach Durchtritt des Blutes durch den Muskel des nephrektomier-. ten Tieres stärker als nach Durchtritt des Blutes durch den normalen Muskel konzentriert ist. Dies dürfte höchstwahrscheinlich dahin aufgefasst werden, dass das durch Nephrektomie herbeigeführte hydrämische Blut beim Durchtritt durch den Muskel sein Wasser mehr als in der Norm in denselben abgibt, so dass die Erhöhung der Hämoglobinkonzentration stärker eintritt.

Serumeiweiss nahm, trotzdem das Blut durch den Einfluss der Nephrektomie eine Verdünnung erfuhr, auf der arteriellen und venösen Seite eher zu. Die Zunahme des Serumeiweisses war in 6 Versuchen ohne Ausnahme auf der venösen Seite mehr oder weniger grösser. Dem Durchschnittswert nach beurteilt, stieg der Serumeiweisswert, der vor Ausführung der Nephrektomie im Arterienblut 7,91\% gewesen war, nach diesem Eingriff auf 8,70\% an, die prozentische Zunahme betrug also $10,0 \%$. Im Venenblut erhöhte sich der Serumeiweisswert, der vor Nephrektomie bei 8,00\% gelegen war, auf 8,91\%, es erfolgte hier eine Zunahme von 11,4\%. Die Differenz in der Zunahme zwischen beiden Blutarten war allerdings recht geringfügig, gleichwohl darf diese Tatsache durchaus nicht ausser Acht gelassen werden, weil es hier auf die Ausnahmelosigkeit der Ergebnisse ankommt. Ein Vergleich der Eiweisswerte in beiden Blutarten vor und nach der Nephrektomie ergab: Vor Ausführung der Nephrektomie stieg der Eiweisswert, der vor Durchtritt durch den Gastrocnemius 7,91\% aufgewiesen hatte, nach demselben auf $8,00 \%$ an, die prozentische Zunahme war nur 1,1\%, nach Ausführung der Nephrektomie erhöhte sich der Serumeiweisswert, der vor Durchtritt durch den Muskel zu 8,70\% ermittelt wurde, nach demselben auf 8,91\% an, die Zunahme belief sich also auf 2,4\%. Hieraus geht klar hervor, dass der Muskel des der Nephrektomie unterworfenen Tiers mehr Eiweiss in das zugehörige Blut mobilisiert, als der normale Muskel. Dass dies nicht lediglich durch eine Eindickung des Blutes infolge der Wasserabgabe in den Muskel bedingt ist, erhellt aus der Tatsache, dass gegenüber der Hämoglobinzunahme $(1,7 \%)$ nach Durchtritt des Blutes durch den Muskel die Eiweisszunahme (2,4\%) nach Durchtritt durch den Muskel vergrössert ist.

K. o. D. sank, wie viele eingangs genannte Autoren erkannt haben, nach Ausführung der Nephrektomie ausnahmslos ab und dieses Absinken erwies sich im Venenblut nach Durchtritt durch den Muskel stets als grösser. Zwar sinkt der k.o.D. auch unter normalen Verhältnissen 
Tabelle

Bestimmungen an

\begin{tabular}{|c|c|c|c|c|c|c|c|c|c|c|c|c|c|}
\hline \multirow{2}{*}{ 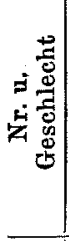 } & \multirow{2}{*}{\multicolumn{2}{|c|}{$\begin{array}{l}\text { Zeit d. } \\
\text { Blutentnahme }\end{array}$}} & \multirow{2}{*}{ 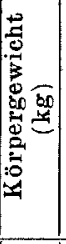 } & \multirow{2}{*}{ 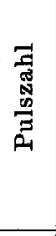 } & \multirow{2}{*}{ 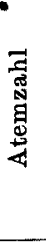 } & \multicolumn{5}{|c|}{ Hämoglobin (g/dl) } & \multicolumn{3}{|c|}{ Serumeiweiss } \\
\hline & & & & & & A & $\begin{array}{l}\text { Diff. } \\
\text { in \% }\end{array}$ & $\mathbf{V}$ & $\begin{array}{l}\text { Diff. } \\
\text { in } \%\end{array}$ & 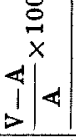 & A & $\begin{array}{l}\text { Diff. } \\
\text { in } \%\end{array}$ & V \\
\hline $\begin{array}{l}1 \\
\delta\end{array}$ & \multicolumn{2}{|c|}{$\begin{array}{l}\text { Vor d. Operation } \\
\text { Nach d. Operation }\end{array}$} & $\begin{array}{l}16,6 \\
17,0\end{array}$ & $\begin{array}{l}64 \\
86\end{array}$ & $\begin{array}{l}20 \\
17\end{array}$ & $\begin{array}{l}13,50 \\
12,80\end{array}$ & $-5,2$ & $\begin{array}{l}13,56 \\
12,97\end{array}$ & $-4,7$ & $\begin{array}{l}+0,4 \\
+1,3\end{array}$ & $\begin{array}{l}8,28 \\
8,75\end{array}$ & $+5,7$ & $\begin{array}{l}8,38 \\
8,97\end{array}$ \\
\hline $\begin{array}{l}2 \\
\delta\end{array}$ & \multicolumn{2}{|c|}{$\begin{array}{l}\text { Vor d. Operation } \\
\text { Nach d. Operation }\end{array}$} & $\begin{array}{l}16,5 \\
15,4\end{array}$ & $\begin{array}{l}54 \\
90\end{array}$ & $\begin{array}{l}17 \\
22\end{array}$ & $\begin{array}{l}14,72 \\
14,28\end{array}$ & $-3,0$ & $\begin{array}{l}14,80 \\
14,58\end{array}$ & $-1,5$ & $\begin{array}{r}+0,5 \\
+2,1\end{array}$ & $\begin{array}{l}8,17 \\
9,10\end{array}$ & $+11,4$ & $\begin{array}{l}8,24 \\
9,29\end{array}$ \\
\hline $\begin{array}{l}3 \\
\text { 古 }\end{array}$ & \multicolumn{2}{|c|}{$\begin{array}{l}\text { Vor d. Operation } \\
\text { Nach d. Operation }\end{array}$} & $\begin{array}{l}24,5 \\
24,8\end{array}$ & $\begin{array}{l}84 \\
90\end{array}$ & $\begin{array}{l}18 \\
22\end{array}$ & $\begin{array}{l}14,38 \\
14,06\end{array}$ & $-2,2$ & $\begin{array}{l}14,44 \\
14,32\end{array}$ & $-0,8$ & $\begin{array}{r}+0,4 \\
+1,8\end{array}$ & $\begin{array}{l}8,28 \\
9,29\end{array}$ & $+12,2$ & $\begin{array}{l}8,38 \\
9,50\end{array}$ \\
\hline $\begin{array}{l}4 \\
8\end{array}$ & \multicolumn{2}{|c|}{$\begin{array}{l}\text { Vord. Operation } \\
\text { Nach d. Operation }\end{array}$} & $\begin{array}{l}22,2 \\
23,0\end{array}$ & $\begin{array}{r}98 \\
100\end{array}$ & $\begin{array}{l}18 \\
30\end{array}$ & $\begin{array}{l}13,56 \\
13,28\end{array}$ & $-2,1$ & $\begin{array}{l}13,61 \\
13,44\end{array}$ & $-1,2$ & $\begin{array}{l}+0,4 \\
+1,2\end{array}$ & $\begin{array}{l}7,24 \\
7,66\end{array}$ & $+5,8$ & $\begin{array}{l}7,33 \\
7,87\end{array}$ \\
\hline $\begin{array}{l}5 \\
\delta\end{array}$ & \multicolumn{2}{|c|}{$\begin{array}{l}\text { Vor d. Operation } \\
\text { Nach d. Operation }\end{array}$} & $\begin{array}{l}17,1 \\
18,0\end{array}$ & $\begin{array}{l}66 \\
94\end{array}$ & $\begin{array}{l}19 \\
23\end{array}$ & $\begin{array}{l}12,32 \\
12,07\end{array}$ & $-2,0$ & $\begin{array}{l}12,38 \\
12,35\end{array}$ & $-0,2$ & $\begin{array}{r}+0,5 \\
+2,6\end{array}$ & $\begin{array}{l}7,55 \\
8,70\end{array}$ & $+15,2$ & $\begin{array}{l}7,63 \\
8,91\end{array}$ \\
\hline $\begin{array}{l}6 \\
\text { 含 }\end{array}$ & \multicolumn{2}{|c|}{$\begin{array}{l}\text { Vor d. Operation } \\
\text { Nach d. Operation }\end{array}$} & $\begin{array}{l}18,3 \\
20,0\end{array}$ & $\begin{array}{l}62 \\
90\end{array}$ & $\begin{array}{l}18 \\
32\end{array}$ & $\begin{array}{l}13,73 \\
13,33\end{array}$ & $-2,9$ & $\begin{array}{l}13,76 \\
13,53\end{array}$ & $-1,7$ & $\begin{array}{r}+0,2 \\
+1,5\end{array}$ & $\begin{array}{l}7,96 \\
8,69\end{array}$ & $+9,2$ & $\begin{array}{l}8,06 \\
8,92\end{array}$ \\
\hline \multicolumn{2}{|c|}{$\begin{array}{l}\text { Druchsch- } \\
\text { nittwert }\end{array}$} & \multicolumn{3}{|c|}{$\begin{array}{l}\text { Vor d. Operation } \\
\text { Nach d. Operation }\end{array}$} & & $\begin{array}{l}13,70 \\
13,30\end{array}$ & $-2,9$ & $\begin{array}{l}13,76 \\
13,53\end{array}$ & $-1,7$ & $\begin{array}{l}+0,4 \\
+1,7\end{array}$ & $\begin{array}{l}7,91 \\
8,70\end{array}$ & $+10,0$ & $\begin{array}{l}8,00 \\
8,91\end{array}$ \\
\hline
\end{tabular}

nach Durchtritt des Blutes durch den Muskel ab, nach erfolgter Nephrektomie indessen war die Erniedrigung des k.o.D. nach Durchtritt durch den Muskel ausnahmslos stärker. Es seien diese Verhältnisse im folgenden in Durchschnittswerten zahlenmässig auseinandergesetzt: Unter normalen Verhältnissen sank der k.o.D., der vor Durchtritt des Blutes durch den Muskel einen Wert von $277 \mathrm{mmH}_{2} \mathrm{O}$ aufgewiesen hatte, nach Durchtritt durch den Muskel auf $274 \mathrm{~mm}$ ab, das Absenken betrug 1,1\%, wohingegen nach Ausführung der Nephrektomie der k. o. D., der vor Durchtritt des Blutes durch den Muskel einen Wert von $263 \mathrm{~mm}$ betrug, nach demselben auf $252 \mathrm{~mm}$ erniedrigt war, die Erniedrigung belief sich 4,2\%. Die k.o.D.-Erniedrigung. war also gegenüber der Norm beträchtlich grösser.

Von vornherein war eine auffallende Erniedrigung des Drucks pro $\%$ verständlich, weil trotz erheblicher Eiweisszunahme durch Nephrektomie der k. o. D. absank. In der Norm erniedrigte sich Druck pro \%, der vor Durchtritt des Blutes durch den Muskel einen Wert von $35,1 \mathrm{~mm}$ 
1.

nephrektomierten Hunden.

\begin{tabular}{|c|c|c|c|c|c|c|c|c|c|c|c|c|c|}
\hline \multicolumn{2}{|l|}{$(\%)$} & \multicolumn{5}{|c|}{ Kolloid-osm. Druck $\left(\mathrm{mmH}_{2} \mathrm{O}\right)$} & \multicolumn{5}{|c|}{ K.o.D. pro $\%\left(\mathrm{mmH}_{2} \mathrm{O}\right)$} & \multirow[b]{2}{*}{ A } & \multirow[b]{2}{*}{$\begin{array}{l}\text { Diff. } \\
\text { in } \%\end{array}$} \\
\hline $\begin{array}{l}\text { Diff. } \\
\text { in } \%\end{array}$ & 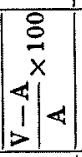 & $\mathbf{A}$ & $\begin{array}{l}\text { Diff. } \\
\text { in } \%\end{array}$ & $\mathrm{~V}$ & $\begin{array}{l}\text { Diff. } \\
\text { in } \%\end{array}$ & $\left|\begin{array}{c}8 \\
\bar{x} \\
4 \\
1 \\
1\end{array}\right|$ & A & $\begin{array}{l}\text { Diff. } \\
\text { in } \%\end{array}$ & $\mathrm{v}$ & $\begin{array}{l}\text { Diff. } \\
\text { in } \%\end{array}$ & 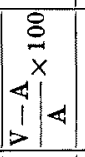 & & \\
\hline $\begin{array}{r} \\
+\quad 6,9\end{array}$ & $\left|\begin{array}{l}+1,2 \\
+2,4\end{array}\right|$ & $\begin{array}{l}283 \\
277\end{array}$ & $-2,1$ & $\begin{array}{l}277 \\
270\end{array}$ & $-2,5$ & $\mid \begin{array}{r}-2,1 \\
-2,5 \\
\end{array}$ & $\begin{array}{l}34,2 \\
31,6\end{array}$ & $-7,6$ & $\begin{array}{l}33,0 \\
30,1\end{array}$ & $-8,8$ & $\left|\begin{array}{l}-3,5 \\
-4,7\end{array}\right|$ & $\begin{array}{l}1,151 \\
1,459\end{array}$ & $+26,7$ \\
\hline$+12,7$ & $\begin{array}{r}+0,8 \\
+2,1\end{array}$ & $\begin{array}{l}291 \\
286\end{array}$ & $-1,7$ & $\begin{array}{l}289 \\
270\end{array}$ & $-6,6$ & $\left|\begin{array}{l}-0,7 \\
-5,6\end{array}\right|$ & $\begin{array}{l}35,6 \\
31,4\end{array}$ & $-11,8$ & $\begin{array}{l}35,1 \\
29,1\end{array}$ & $-17,1$ & $\left|\begin{array}{l}-1,4 \\
-7,3\end{array}\right|$ & $\begin{array}{l}1,061 \\
1,380\end{array}$ & $+30,1$ \\
\hline$+13,4$ & $\begin{array}{l}+1,2 \\
+2,3\end{array}$ & $\begin{array}{l}266 \\
240\end{array}$ & $-9,8$ & $\begin{array}{l}261 \\
228\end{array}$ & $-12,6$ & $\left|\begin{array}{l}-1,9 \\
-5,0\end{array}\right|$ & $\begin{array}{l}32,1 \\
25,8\end{array}$ & $|-19,6|$ & $\begin{array}{l}31,1 \\
24,0\end{array}$ & $-22,8$ & $\begin{array}{r}-3,1 \\
-7,0\end{array} \mid$ & $\begin{array}{l}1,054 \\
1,439\end{array}$ & $+36,5$ \\
\hline$+7,4$ & $\begin{array}{l}+1,2 \\
+2,7\end{array}$ & \begin{tabular}{|l}
273 \\
266 \\
\end{tabular} & $-2,6$ & $\begin{array}{l}271 \\
260\end{array}$ & $-4,0$ & $\left|\begin{array}{l}-0,7 \\
-2,2\end{array}\right|$ & $\begin{array}{l}37,7 \\
34,7\end{array}$ & $-7,9$ & $\begin{array}{l}37,0 \\
33,0\end{array}$ & $-10,8$ & $\left|\begin{array}{r}-1,8 \\
-4,9\end{array}\right|$ & $\begin{array}{l}1,056 \\
1,343\end{array}$ & $+27,2$ \\
\hline$+16,8$ & $\begin{array}{l}+1,0 \\
+2,4\end{array}$ & $\begin{array}{l}273 \\
255\end{array}$ & $-6,6$ & $\begin{array}{l}270 \\
239\end{array}$ & $-11,5$ & $\left|\begin{array}{l}-1,1 \\
-6,3\end{array}\right|$ & $\begin{array}{l}36,1 \\
29,3\end{array}$ & $\mid-18,8$ & $\begin{array}{l}35,4 \\
26,8\end{array}$ & $|-24,3|$ & $\left|\begin{array}{l}-1,9 \\
-8,5\end{array}\right|$ & $\left|\begin{array}{l}1,074 \\
1,405\end{array}\right|$ & $+30,8$ \\
\hline$+10,7$ & $\left|\begin{array}{r}+1,2 \\
+2,6\end{array}\right|$ & $\begin{array}{l}277 \\
255\end{array}$ & $-7,9$ & $\begin{array}{l}274 \\
243\end{array}$ & 一 11,3 & $\left|\begin{array}{l}-1,1 \\
-4,7\end{array}\right|$ & $\begin{array}{l}34,8 \\
29,3\end{array}$ & $-15,8$ & $\begin{array}{l}34,0 \\
27,2\end{array}$ & $-20,3$ & $\begin{array}{l}-2,3 \\
-7,2\end{array} \mid$ & $\begin{array}{l}1,079 \\
1,405\end{array}$ & $+30,2$ \\
\hline$+11,4$ & $\left|\begin{array}{l}+1,1 \\
+2,4\end{array}\right|$ & $\begin{array}{l}277 \\
263\end{array}$ & $-5,0$ & $\begin{array}{l}274 \\
252\end{array}$ & $-8,0$ & $\begin{array}{l}-1,1 \\
-4,2\end{array} \mid$ & $\begin{array}{l}35,1 \\
30,3\end{array}$ & $-13,7$ & $\begin{array}{l}34,3 \\
28,4\end{array}$ & $-17,2$ & $\left|\begin{array}{l}-2,3 \\
-6,3\end{array}\right|$ & $\begin{array}{l}1,079 \\
1,405\end{array}$ & $+30,2$ \\
\hline
\end{tabular}

aufgewiesen hatte, nach demselben auf $34,3 \mathrm{~mm}$, es erfolgte nämlich nur eine Erniedrigung von 2,3\%. Nach Ausführung der Nephrektomie fiel Druck pro \%, der vor Durchtritt durch den Muskel einen Wert von $30,3 \mathrm{~mm}$ aufgewiesen hatte, nach demselben auf $28,4 \mathrm{~mm}$ ab, die Erniedrigung betrug $6,3 \%$. Hieraus ist ersichtlich, dass auch Druck pro \% beim Blut des nephrektomierten Tiers nach Durchtritt durch den Muskel eine grössere Erniedrigung erfährt.

Obige Daten lassen sich kurz dahin zusammenfassen : Nach Durchtritt des Blutes durch den Muskel des nephrektomierten Tiers ist das Hämoglobin im Vergleich mit demselben nach Durchtritt des Blutes durch den normalen Muskel erheblich höher konzentriert, wobei auch das Bluteiweiss in grösserem Masse zunimmt, und der k. o.D. und Druck pro \% stärkere Erniedrigung erfährt. Mit anderen Worten, die Muskulatur, die durch den Funktionsausfall der Niere indirekt geschädigt ist, nimmt aus dem Blut Wasser und gibt ins Blut ihr. Depoteiweiss ab, welches weniger osmoaktiver, grobdisperser Natur ist, was alles ingrös- 
Tbelle 1. Fortsetzung

\begin{tabular}{|c|c|c|c|c|c|c|c|c|c|c|c|c|c|}
\hline \multicolumn{14}{|c|}{ Serum-N } \\
\hline \multicolumn{3}{|c|}{ Gesamt-N $(\mathrm{g} / \mathrm{dl})$} & \multicolumn{5}{|c|}{ Rest-N (g/d1) } & \multicolumn{5}{|c|}{ Albumin-N $(\mathrm{g} / \mathrm{dl})$} & \multirow[b]{2}{*}{$\mathbf{A}$} \\
\hline V & $\begin{array}{l}\text { Diff. } \\
\text { in } \%\end{array}$ & $\left|\begin{array}{c}8 \\
\frac{8}{x} \\
4 \\
1 \\
\end{array}\right|$ & $\mathbf{A}$ & $\begin{array}{l}\text { Diff. } \\
\text { in } \%\end{array}$ & $\mathrm{~V}$ & $\begin{array}{l}\text { Diff. } \\
\text { in } \% 6\end{array}$ & $\left|\begin{array}{c}8 \\
x \\
4 \\
1 \\
2\end{array}\right|$ & $\mathbf{A}$ & $\begin{array}{l}\text { Diff. } \\
\text { in } \%\end{array}$ & $\mathrm{~V}$ & \begin{tabular}{|l|} 
Diff. \\
in $\%$ \\
\end{tabular} & $\left|\begin{array}{c}8 \\
\frac{8}{x} \\
4 \\
1\end{array}\right|$ & \\
\hline $\begin{array}{l}1,163 \\
1,504\end{array}$ & $+29,3$ & $\mid \begin{array}{l}+1,0 \\
+3,1\end{array}$ & $\begin{array}{l}0,028 \\
0,277\end{array} \mid$ & $+889,3$ & $\begin{array}{l}0,029 \\
0,291\end{array}$ & $+903,4$ & $\mid \begin{array}{l}+3,6 \\
+5,0\end{array}$ & $\begin{array}{l}0,634 \\
0,517\end{array} \mid$ & $-18,4$ & $\begin{array}{l}0,625 \\
0,504\end{array}$ & $-19,4$ & $\left|\begin{array}{l}-1,4 \\
-2,5\end{array}\right|$ & $\begin{array}{l}0,489 \\
0,665\end{array}$ \\
\hline $\begin{array}{l}1,078 \\
1,431\end{array}$ & $+32,7$ & $\begin{array}{l}+1,6 \\
+3,7\end{array}$ & $\left|\begin{array}{l}0,029 \\
0,268\end{array}\right|$ & $+824,1$ & $\begin{array}{l}0,031 \\
0,289\end{array}$ & $+832,2$ & $\begin{array}{l}+6,9 \\
+7,8\end{array}$ & $\begin{array}{l}0,559 \\
0,485\end{array}$ & $|-13,2|$ & $\begin{array}{l}0,548 \\
0,472\end{array}$ & $-13,9$ & $\left|\begin{array}{l}-2,0 \\
-2,7\end{array}\right|$ & $\begin{array}{l}0,473 \\
0,627\end{array}$ \\
\hline $\begin{array}{l}1,077 \\
1,484\end{array}$ & $+37,8$ & $\begin{array}{l}+2,2 \\
+3,1\end{array}$ & $\begin{array}{l}0,025 \\
0,243\end{array}$ & +872 & $\begin{array}{l}0,026 \\
0,253\end{array}$ & $+873,1$ & $\left|\begin{array}{l}+4,0 \\
+4,1\end{array}\right|$ & $\begin{array}{l}0,605 \\
0,594\end{array}$ & $-1,8$ & $\begin{array}{c}0,612 \\
0,577\end{array}$ & $-5,7$ & $\left|\begin{array}{l}-1,1 \\
-2,9\end{array}\right|$ & $\begin{array}{l}0,424 \\
0,602\end{array}$ \\
\hline $\begin{array}{l}1,075 \\
1,398\end{array}$ & $+30,0$ & $\begin{array}{r}+1,8 \\
+4,1\end{array}$ & $\left|\begin{array}{l}0,026 \\
0,254\end{array}\right|$ & $+876,9$ & $\left|\begin{array}{l}0,028 \\
0,277\end{array}\right|$ & $+889,3$ & $\begin{array}{r}+7,7 \\
+9,0\end{array}$ & $\begin{array}{l}0,565 \\
0,491\end{array}$ & $-13,1$ & $\begin{array}{l}0,553 \\
0,473\end{array}$ & $-14,5$ & $\left|\begin{array}{l}-2,1 \\
-3,7\end{array}\right|$ & $\begin{array}{l}0,465 \\
0,598\end{array}$ \\
\hline $\begin{array}{l}1,086 \\
1,454\end{array}$ & $+33,9$ & $\left|\begin{array}{l}+1,1 \\
+3,5\end{array}\right|$ & $\begin{array}{l}0,030 \\
0,287\end{array}$ & $+856,7$ & $\left|\begin{array}{l}0,032 \\
0,307\end{array}\right|$ & $+859,4$ & $\mid \begin{array}{r}+6,7 \\
+7,0\end{array}$ & $\begin{array}{l}0,664 \\
0,522\end{array}$ & $-21,4$ & $\begin{array}{l}0,662 \\
0,506\end{array}$ & $-23,6$ & $\mid \begin{array}{r}-0,3 \\
-3,1\end{array}$ & $\begin{array}{l}0,380 \\
0,596\end{array}$ \\
\hline $\begin{array}{l}1,096 \\
1,452\end{array}$ & $+32,5$ & $\begin{array}{l}+1,6 \\
+3,3\end{array}$ & $\begin{array}{l}0,028 \\
0,268\end{array}$ & $+857,1$ & $\begin{array}{l}0,029 \\
0,285\end{array}$ & $+882,7$ & $\begin{array}{r}+3,6 \\
+6,3\end{array}$ & $\begin{array}{l}0,605 \\
0,519\end{array}$ & $-14,2$ & $\begin{array}{l}0,600 \\
0,503\end{array}$ & $-16,2$ & $\mid \begin{array}{r}-0,8 \\
-3,1\end{array}$ & $\begin{array}{l}0,446 \\
0,618\end{array}$ \\
\hline $\begin{array}{l}1,096 \\
1,454\end{array}$ & $+32,7$ & $\begin{array}{l}+1,6 \\
+3,5\end{array}$ & $\begin{array}{l}0,028 \\
0,266\end{array}$ & +850 & $\begin{array}{l}0,029 \\
0,284\end{array}$ & $+879,3$ & $\begin{array}{r}+3,6 \\
+6,8\end{array}$ & $\begin{array}{l}0,605 \\
0,521\end{array}$ & $-13,9$ & $\begin{array}{l}0,600 \\
0,506\end{array}$ & $-15,7$ & $\begin{array}{l}-0,8 \\
-2,9\end{array}$ & $\begin{array}{l}0,446 \\
0,618\end{array}$ \\
\hline
\end{tabular}

serem Masse als in der Norm vor sich geht.

Was die Ergebnisse derN-Analysen anbetrifft, nehmen nach Nephrektomie sowohl auf der arteriellen wie auch auf der venösen Seite Gesamt-N, Rest-N und Globulin-N zu, nur Albumin-N allein ab. Die Grade der Zunahme oder Abnahme sind auf der venösen Seite stets grösser. Wenn man obige Daten im einzelnen dem Durchschnittswerte nach betracht, so ergeben sich folgende Zifferzahlen. Gesamt-N, der vor Ausführung der Nephrektomie im Arterienblut zu 1,079 g/dl ermittelt worden war, betrug im Venenblut, das den Muskel durchflossen hat, $1,096 \mathrm{~g} / \mathrm{dl}$, die prozentische Zunahme war nur 1,6\%. Nach Ausführung der Nephrektomie stieg der Gesamt-N, der im Arterienblut, das den Muskel noch nicht durchpassiert hat, zu 1,405 g/dl ermittelt worden war, im Venenblut nach Durchtritt durch den Muskel auf 1,454 g/dl an, die prozentische Zunahme belief sich auf 3,5\%. Rest-N, der im Arterienblut vor Ausführung der Nephrektomie einen Wert von $0,028 \mathrm{~g} / \mathrm{dl}$ gezeigt hatte, stieg im Venenblut nach Durchtritt durch den Muskel 


\begin{tabular}{|c|c|c|c|c|c|c|c|c|c|}
\hline \multicolumn{4}{|c|}{ Globulin-N (g/d1) } & \multicolumn{5}{|c|}{ Albumin/Globulin } & \multirow[b]{2}{*}{ Bemerkungen. } \\
\hline $\begin{array}{l}\text { Diff. } \\
\text { in } \%\end{array}$ & V & $\begin{array}{l}\text { Diff. } \\
\text { in } \%\end{array}$ & $\left|\begin{array}{c}8 \\
0 \\
x \\
1 \\
1\end{array}\right| 4$ & $\mathbf{A}$ & $\begin{array}{l}\text { Diff. } \\
\text { in } \%\end{array}$ & $\mathrm{v}$ & \begin{tabular}{|l|} 
Diff. \\
in $\%$
\end{tabular} & 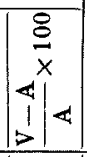 & \\
\hline$+36,0$ & $\begin{array}{l}0,509 \\
0,709 \\
\end{array}$ & $+39,3$ & $\left|\begin{array}{l}+4,1 \\
+6,6\end{array}\right|$ & $\begin{array}{l}1,30 \\
0,78\end{array}$ & $-40,0$ & $\begin{array}{l}1,23 \\
0,71\end{array}$ & $-42,3$ & $\left|\begin{array}{l}-5,4 \\
-9,0\end{array}\right|$ & 69 Std. nach Nephrektomie. \\
\hline$+32,5$ & $\begin{array}{l}0,449 \\
0,670\end{array}$ & $+34,3$ & $\begin{array}{l}+5,5 \\
+6,8\end{array}$ & $\begin{array}{l}1,18 \\
0,77\end{array}$ & $-34,7$ & $\begin{array}{l}1,10 \\
0,70\end{array}$ & $-36,4$ & $\left|\begin{array}{l}-6,8 \\
-9,1\end{array}\right|$ & 55 Std. nach Nephrektomie. \\
\hline$+42,0$ & $\left|\begin{array}{l}0,439 \\
0,644\end{array}\right|$ & $+46,7$ & $\left|\begin{array}{l}+3,5 \\
+7,0\end{array}\right|$ & $\begin{array}{l}1,43 \\
0,99\end{array}$ & $-30,8 \mid$ & $\begin{array}{l}1,39 \\
0,89\end{array}$ & $-36,0$ & $\left|\begin{array}{r}-2,8 \\
-10,1\end{array}\right|$ & 48 Std. nach Nephrektomie. \\
\hline$+28,6$ & $\begin{array}{l}0,494 \\
0,648\end{array}$ & $+31,2$ & $\left|\begin{array}{l}+6,2 \\
+8,4\end{array}\right|$ & $\begin{array}{l}1,21 \\
0,82\end{array}$ & $-32,2$ & $\begin{array}{l}1,12 \\
0.73\end{array}$ & $-34,8$ & $\left|\begin{array}{l}-7,4 \\
-11,0\end{array}\right|$ & 55 Std. nach Nephrektomie. \\
\hline$+56,8$ & 0,392 & $+63,5$ & $\left|\begin{array}{l}+3,1 \\
+7,5\end{array}\right|$ & $\begin{array}{l}1,75 \\
0,87 \\
\end{array}$ & $-50,3$ & $\begin{array}{l}1,69 \\
0,79 \\
\end{array}$ & $-53,2$ & $\left|\begin{array}{l}-3,4 \\
-9,2\end{array}\right|$ & 55 Std. wach Nephrektomie. \\
\hline$+38,6$ & $\left|\begin{array}{l}0,464 \\
0,664\end{array}\right|$ & $+43,1$ & $\left|\begin{array}{r}+4,0 \\
+7,4\end{array}\right|$ & $\begin{array}{l}1,36 \\
0,84\end{array}$ & $-38,2$ & $\begin{array}{l}1,29 \\
0,76\end{array}$ & $-41,1$ & $\left|\begin{array}{l}-5,1 \\
-9,5\end{array}\right|$ & 72 Std. nach Nephrektomie. \\
\hline$+38,6$ & $\begin{array}{l}0,466 \\
0,663\end{array}$ & $+42,3$ & $\begin{array}{r}+4,5 \\
+7,3\end{array}$ & $\begin{array}{l}1,37 \\
0,84\end{array}$ & $-38,7 \mid$ & $\begin{array}{l}1,30 \\
0,76\end{array}$ & $-41,5$ & $\left|\begin{array}{l}-5,1 \\
-9,5\end{array}\right|$ & \\
\hline
\end{tabular}

auf $0,029 \mathrm{~g} / \mathrm{dl}$, es erfolgte hier eine recht spärliche Zunahme von 3,6\%. Nach Ausführung der Nephrektomie war ein Wert von $0,266 \mathrm{~g} / \mathrm{dl}$ vor Durchtritt durch den Muskel nach demselben auf 0,284 g/dl erhöht, hier erfolgte eine Zunahme von $6,8 \%$, die beinahe das zweifache der in der Norm aufgetreten Zunahme erreichte. Es lässt sich also sagen, dass im Blut, das den durch Nephrektomie geschädigten Muskel durchflossen hat, Gesamt-N sowie Rest- $\mathrm{N}$ ausserordentlich vermehrt gefunden werden.

Albumin, welches vor Ausführung der Nephrektomie im Arterienblut vor Durchtritt durch den Muskel zu 0,605 g/dl beziffert worden war, fiel im Venenblut nach demselben auf $0,600 \mathrm{~g} / \mathrm{dl} \mathrm{ab}$, die prozentische $\mathrm{Ab}$ nahme betrug also 0,8\%. Nach Ausführung der Nephrektomie nahm das Albumin, welches im Arterienblut einen Wert von $0,521 \mathrm{~g} / \mathrm{dl}$ aufgewiesen hatte, im Venenblut auf $0,506 \mathrm{~g} / \mathrm{dl}$. ab, die prozentische Abnahme betrug 2,9\%. Es resultierte also gegenüber der Abnahme nach Durchtritt durch den normalen Muskel hier eine erhebliche Albuminab- 
nahme.

Globulin, das vor Ausführung der Nephrektomie im Arterienblut einen Wert von $0,446 \mathrm{~g} / \mathrm{dl}$ aufwies, wurde im Venenblut zu 0,466 g/dl ermittelt, die prozentische Zunahme betrug 4,5\%. Nach Ausführung der Nephrektomie wurde das Globulin im Arterienblut zu 0,618 g/dl, im Venenblut zu 0,663 g/dl ermittelt, die prozentische Zunahme betrug 7,3 $\%$. Es hat sich nämlich herausgestellt, dass das Globulin, im Gegensatz zum Albumin, nach Durchtritt des Blutes durch den Muskel in höherem Masse als nach demselben durch den normalen Muskel zunimmt. Dieses Ergebnis der N-Analyse stimmt mit Bestimmungen des k.o.D. gut überein. Mithin erweist sich der Albumin-Globulin-Quotient im Venenblut nach Durchtritt durch den Muskel nach Nephrektomie als ausserordentlich klein.

Aus obigen Ergebnissen kann erschlossen werden, dass beim Hunde, der durch die Nephrektomie urämische Symptome darbietet, der Skelettmuskel auf das Blut, das den Muskel durchfliesst, folgende Einflüsse ausübt: 1. Der Muskel entnimmt dem Blut Wasser und bringt dasselbe zur Eindickung, 2. gibt Eiweiss, besonders die Eiweisskörper von Globulinfraktionen ins Blut ab, 3. bewirkt die Erniedrigungen von k. o.D. sowie Druck pro \%, 4. mobilisiert den Rest-N ins Blut.

Obige Veränderungen sind zwar auch beim Muskel in der Norm anzutrefen, treten aber bei der Nephrektomie in beträchtlich höherem Masse auf.

\section{Versuchan Hunden mit Ureterenunter- bindung.}

Unter Anwendung der 7 gesunden Hunde wurden zuerst an dem M. gastrocnemius auf der einen Seite Bestimmungen von Blutdaten ausgeführt und dann wurde jedes einzelne Versuchstier der beiderseitigen Ureterenunterbindung unterworfen, nach Ablauf von 55-70 Stunden wurden an dem anderseitigen Gastrocnemius Parallelbestimmungen ausgeführt (Tab. 2).

Hämoglobin erfuhr durch beiderseitige Ureterenunterbindung sowohl auf der arteriellen wie auch auf der venösen Seite ebenfalls eine Verdünnung, der Grad der Verdünnung war aber auf der venösen Seite ohne Ausnahme geringer. Während auf der arteriellen Seite die Differenz in Prozenten sich auf durchschnittlich -4,7 bezifferte, betrug sie auf der venösen Seite -2,3. Dies läuft darauf hinaus, dass die Hämoglobinkonzentration nach Durchtritt durch den Muskel erhöht ist. Vor Ureterenunterbindung betrug der Hämoglobinwert im Durchschnitt im 
Arterienblut 14,76 g/dl, im Venenblut 14,87 g/dl, der Grad der.Konzentrierung war $0,7 \%$. Nach Ureterenunterbindung betrug der Hämoglobinwert im Arterienblut 14,07 g/dl, im Venenblut 14,53 g/dl, die Erhöhung der Konzentration beziffert sich auf 3,3\%. Hieraus geht hervor, dass die Hämoglobinkonzentration nach Durchtritt des Blutes durch den Muskel des der Ureterenunterbindung unterworfenen Tiers im stärkeren Masse als nach demselben durch den normalen Muskel erhöht ist. Die Beziehung gestaltet sich in eben gleicher Weise wie bei der Nephrektomie.

Serumeiweiss erfährt, trotzdem das Blut durch die Ureterenunterbindung eine Verdünnung aufweist, im arteriellen und venösen Blut eher eine Zunahme. Die Zunahine im Venenblut war in 7 Versuchen ohne Ausnahme ein wenig höher.

Serumeiweiss im Arterienblut nämlich, welches vor Ureterenunterbindung im Mittel einen Wert von 7,56\% aufwies, stieg nach Ureterenunterbindung auf $8,53 \%$ an, die prozentische Zunahme war $12,8 \%$. Serumeiweiss im Venenblut, welches vor Ureterenunterbindung zu 7,67 $\%$ ermittelt wurde, nahm nach diesem Eingriff auf $8,82 \% \mathrm{zu}$, die $\mathrm{Zu}$ nahme betrug 15,0\%. Obwohl die Differenz in der Zunahme, wie oben erwähnt, recht geringfügig ist, ist sie wegen der Ausnahmelosigkeit beobachtenswert.

Wenn man nun Arterienwerte und Venenwerte für Serumeiweiss vor und nach Ureterenunterbindung gegeneinander vergleicht, so ergibtsich folgendes: Vor Ureterenunterbindung stieg der Serumeiweissgehalt, der vor dem Einströmen in den Gastrocnemius 7,56\% betrug, nach Durchtritt durch denselben auf 7,67\% an, diese Erhöhung bezifferte sich nur auf 1,4\%. Nach Ureterenunterbindung wurde ein Wert von $8,53 \%$ vor Durchtritt durch den Muskel ermittelt, diese Wert wurde nach demselben auf 8,82\% erhöht, die prozentische Erhöhung nach Durchtritt durch den Muskel betrug 3,4\%. Die Eiweisszunahme, welche nach Ureterenunterbindung nach Durchtritt des Blutes durch den Muskel erfolgte, war unverkennbar grösser als die nach Durchtritt des Blutes durch den normalen Muskel; die prozentische Zunahme war aber 3,4\%, diese Zahl war, verglichen mit der Hämoglobinzunahme von $3,3 \%$, annähernd dieselbe. Auch einzelnen Werten nach kann man zwischen Hämoglobinzunahme und Serumeiweisszunahme (siehe unter Kolumne $\frac{\mathrm{V}-\mathrm{A}}{\mathrm{A}} \times 100 \mathrm{in} \mathrm{Tab.2)}$ keine scharfe Grenze ziehen. Die Frage deshalb, ob die Zunahme des Bluteiweisses, welche nach Ureterenunterbindung nach Durchtritt des Blutes durch den Muskel erfolgt, auf die Mobilisierung des Eiweisses von seiten des Muskels zurückzuführen sei, oder da 
Tabelle

Bestimmungen an Hunden

\begin{tabular}{|c|c|c|c|c|c|c|c|c|c|c|c|c|}
\hline \multirow{2}{*}{ 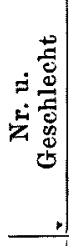 } & \multirow{2}{*}{$\begin{array}{c}\text { Zeit d. } \\
\text { Blutentnahme }\end{array}$} & \multirow{2}{*}{ 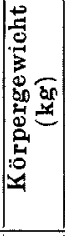 } & \multirow{2}{*}{ 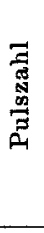 } & \multirow{2}{*}{ 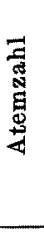 } & \multicolumn{5}{|c|}{ Hämoglobin (g/dl) } & \multicolumn{3}{|c|}{ Serumeiweiss } \\
\hline & & & & & A & $\begin{array}{l}\text { Diff. } \\
\text { in } \%\end{array}$ & V & $\begin{array}{l}\text { Diff. } \\
\text { in } \%\end{array}$ & $\left|\begin{array}{c}8 \\
x \\
x \\
1 \\
1\end{array}\right|$ & $\mathbf{A}$ & $\begin{array}{l}\text { Diff. } \\
\text { in } \%\end{array}$ & V \\
\hline $\begin{array}{l}1 \\
\delta\end{array}$ & $\begin{array}{l}\text { Vor d. Operation } \\
\text { Nach d. Operation }\end{array}$ & $\begin{array}{l}13,4 \\
14,5\end{array}$ & $\begin{array}{l}74 \\
95\end{array}$ & $\begin{array}{l}20 \\
25\end{array}$ & $\begin{array}{l}15,64 \\
14,77\end{array}$ & $-5,6$ & $\begin{array}{l}15,64 \\
15,19\end{array}$ & $-2,9$ & $\begin{array}{l} \pm 0 \\
+2,8\end{array} \mid$ & $\begin{array}{l}7,29 \\
8,71\end{array}$ & $+, 19,5$ & $\begin{array}{l}7,38 \\
8,92\end{array}$ \\
\hline $\begin{array}{l}2 \\
\delta\end{array}$ & $\begin{array}{l}\text { Vor d. Operation } \\
\text { Nach d. Operation }\end{array}$ & $\begin{array}{l}15,6 \\
16,0\end{array}$ & $\begin{array}{l}62 \\
74\end{array}$ & $\begin{array}{l}11 \\
12\end{array}$ & $\begin{array}{l}14,35 \\
13,20\end{array}$ & $-8,0$ & $\begin{array}{l}14,46 \\
13,85\end{array}$ & $-4,2$ & $\begin{array}{l}+0,8 \\
+4,9\end{array}$ & $\begin{array}{l}6,51 \\
7,63\end{array}$ & $+17,2$ & $\begin{array}{l}6,64 \\
7,96\end{array}$ \\
\hline $\begin{array}{l}3 \\
\hat{\delta}\end{array}$ & $\begin{array}{l}\text { Vor d. Operation } \\
\text { Nach d. Operation }\end{array}$ & $\begin{array}{l}19,5 \\
20,0\end{array}$ & $\begin{array}{l}62 \\
94\end{array}$ & $\begin{array}{l}16 \\
34\end{array}$ & $\begin{array}{l}14,19 \\
13,85\end{array}$ & $-2,4$ & $\begin{array}{l}14,24 \\
14,07\end{array}$ & $-1,2$ & $\begin{array}{l}+0,3 \\
+1,6\end{array}$ & $\begin{array}{l}8,30 \\
8,49\end{array}$ & $+2,3$ & $\begin{array}{l}8,39 \\
8,71\end{array}$ \\
\hline $\begin{array}{l}4 \\
\delta\end{array}$ & $\begin{array}{l}\text { Vor d. Operation } \\
\text { Nach d. Operation }\end{array}$ & $\begin{array}{l}18,0 \\
19,4\end{array}$ & $\begin{array}{l}51 \\
56\end{array}$ & $\begin{array}{l}16 \\
22\end{array}$ & $\begin{array}{l}14,97 \\
14,14\end{array}$ & $-5,5$ & $\begin{array}{l}15,11 \\
14,74\end{array}$ & $-2,4$ & $\begin{array}{r}+0,9 \\
+4,2\end{array}$ & $\begin{array}{l}7,55 \\
8,57\end{array}$ & $+13,5$ & $\begin{array}{l}7,65 \\
8,85\end{array}$ \\
\hline $\begin{array}{l}5 \\
\delta\end{array}$ & $\begin{array}{l}\text { Vor d. Operation } \\
\text { Nach d. Operation }\end{array}$ & $\begin{array}{l}20,0 \\
20,5\end{array}$ & $\begin{array}{l}65 \\
84\end{array}$ & $\begin{array}{l}17 \\
25\end{array}$ & $\begin{array}{l}15,29 \\
14,71\end{array}$ & $-3,8$ & $\begin{array}{l}15,44 \\
15,24\end{array}$ & $-1,3$ & $\begin{array}{l}+1,0 \\
+3,6\end{array}$ & $\begin{array}{l}7,47 \\
8,49\end{array}$ & $+13,6$ & $\begin{array}{l}7,58 \\
8,76\end{array}$ \\
\hline $\begin{array}{l}6 \\
\delta\end{array}$ & $\begin{array}{l}\text { Vor d. Operation } \\
\text { Nach d. Operation }\end{array}$ & $\begin{array}{l}25,0 \\
26,0\end{array}$ & $\begin{array}{l}65 \\
82\end{array}$ & $\begin{array}{l}16 \\
21\end{array}$ & $\begin{array}{l}14,24 \\
13,56\end{array}$ & $-4,8$ & $\begin{array}{l}14,42 \\
14,14\end{array}$ & $-1,9$ & $\begin{array}{l}+1,3 \\
+4,3\end{array}$ & $\begin{array}{l}7,81 \\
8,75\end{array}$ & $+12,0$ & $\begin{array}{l}7,93 \\
9,27\end{array}$ \\
\hline $\begin{array}{l}7 \\
\delta\end{array}$ & $\begin{array}{l}\text { Vor d. Operation } \\
\text { Nach d. Operation }\end{array}$ & $\begin{array}{l}18,5 \\
20,0\end{array}$ & $\begin{array}{l}66 \\
85\end{array}$ & $\begin{array}{l}16 \\
30\end{array}$ & $\begin{array}{l}14,63 \\
14,27\end{array}$ & $-2,5$ & $\begin{array}{l}14,80 \\
14,52\end{array}$ & $-1,9$ & $\begin{array}{l}+1,2 \\
+1,7\end{array}$ & $\begin{array}{l}8,02 \\
9,10\end{array}$ & $+13,5$ & $\begin{array}{l}8,10 \\
9,27\end{array}$ \\
\hline $\begin{array}{l}\text { Durchsch- } \\
\text { nittwert }\end{array}$ & $\begin{array}{l}\text { Vor d. Ope } \\
\text { Nach d. Or }\end{array}$ & tio & & & $\begin{array}{l}14,76 \\
14,07\end{array}$ & $-4,7$ & $\begin{array}{l}14,87 \\
14,53\end{array}$ & $-2,3$ & $\begin{array}{l}+0,7 \\
+3,3\end{array}$ & $\begin{array}{l}7,56 \\
8,53\end{array}$ & $+12,8$ & $\begin{array}{l}7,67 \\
8,82\end{array}$ \\
\hline
\end{tabular}

hierbei die prozentische Zunahme mit der Zunahme des Hämoglobins parallel geht, lediglich auf eine Bluteindickung bezogen werden könnte, kann nicht, wie bei der Nephrektomie, ohne weiteres beantwortet werden. Auf diese Frage wird später nach Betrachtung der Ergebnisse der k. o. D.-Bestimmung und N-Analyse eingegangen werden.

K.o.D. sinkt sowohl auf der arteriellen wie auch auf der venösen Seite $a b$, besonders ausgeprägt ist dieses Absinken im venösen Blut, das den Muskel durchflossen hat. Auch in der Norm erfährt der k. o. D. nach Durchtritt durch den Muskel in der Regel eine Erniedrigung, nach Ureterenunterbindung aber geschieht diese ohne Ausnahme in stärkerem Masse. Im Arterienblut vor dem Einströmen in den Muskel beträgt der k.o.D. in der Norm durchschnittlich $289 \mathrm{mmH}_{2} \mathrm{O}$, nach Durchtritt des Blutes durch dem Muskel sinkt er auf $283 \mathrm{~mm}$ ab, es erfolgt ein Absinken von $2,1 \%$. Demgegenüber fällt der k. o. D. nach Ureterenunterbindung, der vor dem Einströmen des Blutes in den Muskel einen durch- 
2.

mit Ureterenunterbindung.

\begin{tabular}{|c|c|c|c|c|c|c|c|c|c|c|c|c|c|}
\hline \multicolumn{2}{|l|}{$(\%)$} & \multicolumn{5}{|c|}{ Kolloid-osm. Druck $\left(\mathrm{mmH}_{2} \mathrm{O}\right)$} & \multicolumn{5}{|c|}{ K.o.D. pro $\%\left(\mathrm{mmH}_{2} \mathrm{O}\right)$} & & \\
\hline $\begin{array}{l}\text { Diff. } \\
\text { in } \%\end{array}$ & $\begin{array}{c}8 \\
0 \\
x \\
1 \\
1 \\
1\end{array}$ & $\mathbf{A}$ & $\begin{array}{l}\text { Diff. } \\
\text { in } \%\end{array}$ & $\mathrm{~V}$ & $\begin{array}{l}\text { Diff. } \\
\text { in } \%\end{array}$ & $\begin{array}{c}8 \\
0 \\
x \\
4 \\
11 \\
>1\end{array}$ & A & $\begin{array}{l}\text { Diff. } \\
\text { in } \%\end{array}$ & V & $\begin{array}{l}\text { Diff. } \\
\text { in } \% \frac{0}{0}\end{array}$ & $\begin{array}{c}g \\
8 \\
x \\
1 \\
1\end{array}$ & A & $\begin{array}{l}\text { Diff. } \\
\text { in } \%\end{array}$ \\
\hline$+20,9$ & $\begin{array}{r}+1,2 \\
+2,4\end{array}$ & $\begin{array}{l}310 \\
304\end{array}$ & $-1,9$ & $\begin{array}{l}296 \\
288\end{array}$ & $-2,7$ & $\begin{array}{l}-4,5 \\
-5,3\end{array}$ & $\begin{array}{l}42,5 \\
34,9\end{array}$ & $-17,9$ & $\begin{array}{l}40,1 \\
32,3\end{array}$ & $-19,4$ & $\begin{array}{l}-5,6 \\
-7,4\end{array} \mid$ & $\begin{array}{l}1,032 \\
1,358\end{array}$ & $+31,6$ \\
\hline$+19,9$ & $\left|\begin{array}{r}+2,0 \\
+4,3\end{array}\right|$ & $\begin{array}{l}260 \\
244\end{array}$ & $-6,1$ & $\begin{array}{l}255 \\
237\end{array}$ & $-7,0$ & $\begin{array}{l}-1,9 \\
-2,9\end{array}$ & $\begin{array}{l}39,9 \\
32,0\end{array}$ & $-19,8$ & $\begin{array}{l}38,4 \\
29,8\end{array}$ & $-22,4$ & $\left|\begin{array}{l}-3,7 \\
-6,9\end{array}\right|$ & $\begin{array}{l}1,032 \\
1,407\end{array}$ & $+36,3$ \\
\hline$+3,8$ & $\begin{array}{l}+1,1 \\
+2,6\end{array}$ & $\begin{array}{l}299 \\
252\end{array}$ & $-15,7$ & $\begin{array}{l}296 \\
244\end{array}$ & $-17,6$ & $\begin{array}{l}-1,0 \\
-3,2\end{array}$ & $\begin{array}{l}36,0 \\
29,7\end{array}$ & $-17,5$ & $\begin{array}{l}35,3 \\
28,0\end{array}$ & $-20,7$ & $\left|\begin{array}{l}-1,9 \\
-5,7\end{array}\right|$ & $\begin{array}{l}0,896 \\
1,156\end{array}$ & $+29,0$ \\
\hline$+15,7$ & $\left|\begin{array}{l}+1,3 \\
+3,3\end{array}\right|$ & $\begin{array}{l}288 \\
271\end{array}$ & $-5,9$ & $\begin{array}{l}282 \\
263\end{array}$ & $-6,7$ & $\begin{array}{l}-2,1 \\
-2,9\end{array}$ & $\begin{array}{l}38,1 \\
31,6\end{array}$ & $-17,1$ & $\begin{array}{l}36,9 \\
29,7\end{array}$ & $-19,5$ & $\begin{array}{l}-3,1 \\
-6,0\end{array}$ & $\begin{array}{l}1,003 \\
1,291\end{array}$ & $+28,7$ \\
\hline$+15,6$ & $\left|\begin{array}{l}+1,5 \\
+3,2\end{array}\right|$ & $\begin{array}{l}287 \\
269\end{array}$ & $-6,3$ & $\begin{array}{l}281 \\
259\end{array}$ & $-7,8$ & $\begin{array}{l}-2,1 \\
-3,7\end{array}$ & $\begin{array}{l}38,4 \\
31,7\end{array}$ & $-17,4$ & $\begin{array}{l}37,1 \\
29,6\end{array}$ & $-20,2$ & $\begin{array}{l}-3,4 \\
-6,6\end{array}$ & $\begin{array}{l}0,998 \\
1,280\end{array}$ & $+28,2$ \\
\hline$+16,9$ & $\mid \begin{array}{l}+1,5 \\
+5,9\end{array}$ & $\begin{array}{l}284 \\
278\end{array}$ & $-2,1$ & $\begin{array}{l}281 \\
273\end{array}$ & $-2,8$ & $\begin{array}{l}-1,0 \\
-1,8\end{array}$ & $\begin{array}{l}36,4 \\
31,8\end{array}$ & $-12,6$ & $\begin{array}{l}35,4 \\
29,4\end{array}$ & $|-16,9|$ & $\begin{array}{l}-2,7 \\
-7,5\end{array}$ & $\begin{array}{l}0,946 \\
1,145\end{array}$ & $+21,0$ \\
\hline$+14,4$ & $\begin{array}{l}+1,0 \\
+1,9\end{array}$ & \begin{tabular}{|l|}
294 \\
288
\end{tabular} & $-2,0$ & $\begin{array}{l}289 \\
282\end{array}$ & $-2,4$ & $\begin{array}{l}-1,7 \\
-2,1\end{array}$ & $\begin{array}{l}36,6 \\
31,6\end{array}$ & $-13,7$ & $\begin{array}{l}35,7 \\
30,4\end{array}$ & $-14,8$ & \begin{tabular}{|}
$-2,4$ \\
$-3,8$ \\
\end{tabular} & $\begin{array}{l}1,037 \\
1,362\end{array}$ & $+31,3$ \\
\hline$+15,0$ & $\begin{array}{r}+1,4 \\
+3,4\end{array}$ & $\begin{array}{l}289 \\
272\end{array}$ & $-5,9$ & $\begin{array}{l}283 \\
264\end{array}$ & $-6,7$ & $\begin{array}{l}-2,1 \\
-2,0\end{array}$ & $\begin{array}{l}38,3 \\
31,9\end{array}$ & $-16,7$ & $\begin{array}{l}37,0 \\
29,9\end{array}$ & $-19,2$ & $\begin{array}{l}-3,4 \\
-6,3\end{array}$ & $\begin{array}{l}0,992 \\
1,285\end{array}$ & $+29,2$ \\
\hline
\end{tabular}

schnittlichen Wert von $272 \mathrm{~mm}$ aufgewiesen hat, nach Durchtritt durch den Muskel auf $264 \mathrm{~mm}$ ab, ein Absinken von 2,9\%. Die prozentische Abnahme des k.o.D. ist also gegenüber der Norm etwas grösser.

Erhebliche Erniedrigung des Drucks pro \% nach der Ureterenunterbindung ist verständlich, weil hier bei der Zunahme des Eiweisses der k.o.D. absinkt. Druck pro \% erniedrigt sich nämlich sowohl im arteriellen als auch im venösen Blut ohne Ausnahme. Unter normalen Verhältnissen fällt der durchschnittliche Wert von $38,3 \mathrm{~mm}$, der vor Durchtritt durch den Muskel ermittelt wird, nach demselben auf 37,0 mm ab, die Abnahme beträgt 3,4\%. Nach Ureterenunterbindung sinkt Druck pro $\%$, der sich vor Durchtritt des Blutes durch den Muskel auf $31,9 \mathrm{~mm}$ beziffert, nach demselben auf $29,9 \mathrm{~mm}$ ab, die Abnahme beträgt 6,3\%. Hier findet sich also gegenüber der Norm eine erhebliche Erniedrigung.

Oben angeführte Ergebnisse lassen sich wie folgt zusammenfas- 
Tbelle 2. Fortsetzung

Serum- $\mathbf{N}$

\begin{tabular}{|c|c|c|c|c|c|c|c|c|c|c|c|c|c|}
\hline \multicolumn{3}{|c|}{ Gesamt-N (g/dl) } & \multicolumn{5}{|c|}{ Rest-N (g/dl) } & \multicolumn{5}{|c|}{ Albumin-N (g/dl) } & \multirow[b]{2}{*}{$\mathbf{A}$} \\
\hline $\mathbf{V}$ & $\begin{array}{l}\text { Diff. } \\
\text { in } \% 0\end{array}$ & $\mid \begin{array}{c}8 \\
0 \\
\times \\
1 \\
>1\end{array}$ & $\mathbf{A}$ & $\begin{array}{l}\text { Diff. } \\
\text { in } \%\end{array}$ & V & $\begin{array}{l}\text { Diff. } \\
\text { in } \%\end{array}$ & 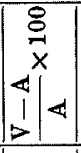 & $\mathbf{A}$ & $\begin{array}{l}\text { Diff. } \\
\text { in } \%\end{array}$ & $\mathbf{V}$ & $\begin{array}{l}\text { Diff. } \\
\text { in } \%\end{array}$ & 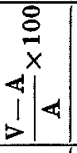 & \\
\hline $\begin{array}{l}1,050 \\
1,397\end{array}$ & $+33,0$ & $\left|\begin{array}{l}+1,7 \\
+2,9\end{array}\right|$ & $\begin{array}{l}0,027 \\
0,276\end{array}$ & $+922,2$ & \begin{tabular}{|l|}
0,028 \\
0,288
\end{tabular} & $+928,6$ & $\begin{array}{r}+3,7 \\
+4,3\end{array}$ & $\begin{array}{l}0,571 \\
0,475\end{array}$ & $-16,8$ & $\begin{array}{l}0,561 \\
0,438\end{array}$ & $-21,9$ & $\left|\begin{array}{l}-1,7 \\
-5,5\end{array}\right|$ & $\begin{array}{l}0,424 \\
0,608\end{array}$ \\
\hline $\begin{array}{l}1,057 \\
1,470\end{array}$ & $+39,1$ & $\left|\begin{array}{r}+2,4 \\
+4,5\end{array}\right|$ & $\begin{array}{l}0,029 \\
0,308\end{array}$ & $+962,1$ & $\begin{array}{l}0,030 \\
0,325\end{array} \mid$ & $+983,3$ & $\left|\begin{array}{r}+3,4 \\
+5,5\end{array}\right|$ & $\begin{array}{l}0,629 \\
0,563\end{array}$ & $-10,5$ & $\begin{array}{l}0,616 \\
0,539\end{array}$ & $-12,5$ & $\begin{array}{l}-2,1 \\
-4,3\end{array}$ & $\begin{array}{l}0,374 \\
0,536\end{array}$ \\
\hline $\begin{array}{l}0,906 \\
1,216\end{array}$ & $+34,2$ & $\begin{array}{l}+1,1 \\
+5,2\end{array} \mid$ & $\begin{array}{l}0,018 \\
0,272\end{array}$ & $+141,1$ & $\left|\begin{array}{l}0,019 \\
0,301\end{array}\right|$ & $+148,4$ & $\begin{array}{r}+5,5 \\
+10,6\end{array} \mid$ & $\begin{array}{l}0,498 \\
0,475\end{array}$ & $-10,5$ & $\left|\begin{array}{l}0,485 \\
0,438\end{array}\right|$ & $-9,7$ & $\begin{array}{r}-2,6 \\
-7,8\end{array}$ & $\begin{array}{l}0,380 \\
0,409\end{array}$ \\
\hline $\begin{array}{l}1,025 \\
1,355\end{array}$ & $+32,2$ & $\left|\begin{array}{r}+2,2 \\
+4,9\end{array}\right|$ & $\begin{array}{l}0,028 \\
0,280\end{array}$ & +900 & $\begin{array}{l}0,029 \\
0,300\end{array}$ & $+934,4$ & $\left|\begin{array}{r}+3,6 \\
+7,1\end{array}\right|$ & $\begin{array}{l}0,578 \\
0,478\end{array}$ & $|-17,3|$ & $\begin{array}{l}0,569 \\
0,439\end{array}$ & $-22,8$ & $\begin{array}{l}-1,5 \\
-8,1\end{array}$ & $\begin{array}{l}0,396 \\
0,532\end{array}$ \\
\hline $\begin{array}{l}1,021 \\
1,368\end{array}$ & $+34,0$ & $\mid \begin{array}{r}+2,3 \\
+6,9\end{array}$ & $\begin{array}{l}0,028 \\
0,280\end{array}$ & +900 & $\begin{array}{l}0,029 \\
0,305\end{array}$ & $+951,7$ & $\begin{array}{r}+3,6 \\
+8,9\end{array}$ & $\begin{array}{l}0,579 \\
0,477\end{array}$ & $-17,6$ & $\begin{array}{l}0,571 \\
0,458\end{array}$ & $-19,8$ & $\begin{array}{l}-1,4 \\
-4,0\end{array}$ & $\begin{array}{l}0,391 \\
0,523\end{array} \mid$ \\
\hline $\begin{array}{l}0,960 \\
1,226\end{array}$ & $+27,7$ & $\left|\begin{array}{l}+1,5 \\
+7,1\end{array}\right|$ & $\begin{array}{l}0,028 \\
0,205\end{array}$ & $+632,1$ & $\begin{array}{l}0,029 \\
0,225\end{array}$ & $+675,9$ & $\begin{array}{r}+3,6 \\
+9,7\end{array}$ & $\begin{array}{l}0,591 \\
0,457\end{array}$ & $-29,3 \mid$ & $\mid \begin{array}{l}0,593 \\
0,456\end{array}$ & $-23,1$ & $\left|\begin{array}{r}+0,3 \\
-2,2\end{array}\right|$ & $\begin{array}{l}0,327 \\
0,483\end{array}$ \\
\hline $\begin{array}{l}1,048 \\
1,398\end{array}$ & $+33,4$ & $\left|\begin{array}{l}+1,1 \\
+2,6\end{array}\right|$ & $\begin{array}{l}0,033 \\
0,287\end{array}$ & $+769,7$ & $\begin{array}{l}0,035 \\
0,307\end{array}$ & $+777,1$ & $\begin{array}{r}+6,1 \\
+7,0\end{array} \mid$ & $\begin{array}{l}0,569 \\
0,508\end{array}$ & $-10,7$ & $\begin{array}{l}0,561 \\
0,459\end{array}$ & $-18,2$ & $\begin{array}{r}-1,4 \\
-9,6\end{array}$ & $\begin{array}{l}0,435 \\
0,567 \\
\end{array}$ \\
\hline $\begin{array}{l}1,009 \\
1,347\end{array}$ & $+33,5$ & $\begin{array}{l}+1,7 \\
+4,8\end{array}$ & $\begin{array}{l}0,027 \\
0,272\end{array}$ & $+907,4$ & $\mid \begin{array}{l}0,028 \\
0,293\end{array}$ & $+946,4$ & $\begin{array}{l}+3,7 \\
+7,7\end{array}$ & $\begin{array}{l}0,571 \\
0,490\end{array}$ & $|-14,2|$ & $\begin{array}{l}0,565 \\
0,461\end{array}$ & $-18,4$ & $\begin{array}{l}-1,0 \\
-5,9\end{array}$ & $\begin{array}{l}0,389 \\
0,522\end{array}$ \\
\hline
\end{tabular}

sen: Nach Ureterenunterbindung, nach Durchtritt des Blutes durch den Muskel, nehmen Hämoglobin und Eiweiss, im Vergleich mit entsprechenden Werten nach Durchtritt des Blutes durch den normalen Muskel, in erheblichem Masse zu, und der k. o.D. sowie Druck pro \% stärker ab. Oben wurde erwähnt, dass die Beantwortung der Frage, woher die Zunahme des Bluteiweisses nach Durchtritt des Blutes durch den Muskel komme, bis zur Betrachtung der Ergebnisse der k. o. D.-Bestimmung und dergleichen vorbehalten wird, weil aus dem Parallelgehen dieser Eiweisszunahme mit der Hämoglobinzunahme angenommen werden dürfte, ob nicht diese Eiweisszunahme lediglich auf eine Bluteindickung bezogen werden könnte. Aus dem Verhalten des k.o.D. gegenüber dem Bluteiweiss und Hämoglobin dürfte man nunmehr vollends darüber ins klare kommen, dass es sich hierbei keineswegs um die Bluteindickung handelt. Denn wenn die Bluteiweisszunahme durch die blosse Bluteindickung zustande käme, müsste dann der k. o.D. nach 


\begin{tabular}{|c|c|c|c|c|c|c|c|c|c|}
\hline \multicolumn{4}{|c|}{ Globulin-N (g-dl) } & \multicolumn{5}{|c|}{ Albumin/Globulin } & \multirow[b]{2}{*}{ Bemerkungen. } \\
\hline $\begin{array}{l}\text { Diff. } \\
\text { in } \%\end{array}$ & $\mathbf{V}$ & $\begin{array}{l}\text { Diff. } \\
\text { in \% }\end{array}$ & $\left|\begin{array}{c}8 \\
8 \\
x \\
1 \\
1 \\
1\end{array}\right| 4$ & $\mathbf{A}$ & $\begin{array}{l}\text { Diff. } \\
\text { in } \%\end{array}$ & $\mathrm{~V}$ & Diff. & 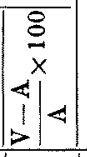 & \\
\hline$+43,4$ & $\begin{array}{l}0,461 \\
0,671\end{array}$ & $+45,5$ & $\begin{array}{r}+8,7 \\
+10,4\end{array}$ & $\begin{array}{l}1,35 \\
0,78\end{array}$ & $-42,2$ & $\begin{array}{l}1,22 \\
0,65\end{array}$ & $-46,7$ & $\left|\begin{array}{r}-9,6 \\
-16,7\end{array}\right|$ & $\left\{\begin{array}{l}70 \text { Std. nach bds. Ureteren- } \\
\text { unterbindung. }\end{array}\right.$ \\
\hline$+43,3$ & $\begin{array}{l}0,411 \\
0,606\end{array}$ & $+47,4$ & $\begin{array}{r}+9,9 \\
+17,8\end{array}$ & $\begin{array}{l}1,68 \\
1,05\end{array}$ & $-37,4$ & $\begin{array}{l}1,50 \\
0,89\end{array}$ & $-40,7$ & $\begin{array}{l}-10,7 \\
-15,2\end{array}$ & $\left\{\begin{array}{l}69 \text { Std. nach bds. Ureteren- } \\
\text { unterbindung. }\end{array}\right.$ \\
\hline$+7,6$ & $\begin{array}{l}0,402 \\
0,477\end{array}$ & $+18,6$ & $\begin{array}{r}+5,8 \\
+16,6\end{array}$ & $\begin{array}{l}1,31 \\
1,16\end{array}$ & $-11,4$ & $\begin{array}{l}1,21 \\
0,92\end{array}$ & $-24,0$ & $\left|\begin{array}{r}-7,6 \\
-20,7\end{array}\right|$ & $\left\{\begin{array}{l}55 \text { Std. naeh bds. Ureteren- } \\
\text { unterbindung. }\end{array}\right.$ \\
\hline$+34,3$ & $\begin{array}{l}0,425 \\
0,614\end{array}$ & $+44,5$ & $\begin{array}{l}+7,3 \\
+15,4\end{array}$ & $\begin{array}{l}1,47 \\
0,92\end{array}$ & $-37,4$ & $\begin{array}{l}1,36 \\
0,73\end{array}$ & $-46,3$ & $\begin{array}{l}-7,5 \\
-20,6\end{array}$ & $\left\{\begin{array}{l}68 \text { Std. nach bds. Ureteren- } \\
\text { unterbindung. }\end{array}\right.$ \\
\hline$+33,7$ & 0,413 & $+41,9$ & $\begin{array}{r}+5,6 \\
+12,0\end{array}$ & $\begin{array}{l}1,48 \\
0,91\end{array}$ & $-38,5$ & $\begin{array}{l}1,38 \\
0,78\end{array}$ & $-43,5$ & $\mid \begin{array}{r}-6,7 \\
-14,3\end{array}$ & $\left\{\begin{array}{l}55 \text { Std. nach bds. Ureteren- } \\
\text { unterbindung. }\end{array}\right.$ \\
\hline$+47,7$ & $\begin{array}{l}0,338 \\
0,545\end{array}$ & $+61,2$ & $\begin{array}{r}+3,4 \\
+12,8\end{array}$ & $\begin{array}{l}1,81 \\
0,95\end{array}$ & $-47,5$ & $\begin{array}{l}1,75 \\
0,84\end{array}$ & $-52,0$ & $\left|\begin{array}{r}-3,3 \\
-11,5\end{array}\right|$ & $\left\{\begin{array}{l}55 \text { Std. nach bds. Ureteren- } \\
\text { unterbindung. }\end{array}\right.$ \\
\hline$+30,3$ & $\begin{array}{l}0,452 \\
0,632\end{array}$ & $+39,8$ & $\begin{array}{r}+3,9 \\
+11,5\end{array}$ & $\begin{array}{l}1,31 \\
0,89\end{array}$ & $-32,1$ & $\begin{array}{l}1,24 \\
0,73\end{array}$ & $-41,1$ & $\left|\begin{array}{r}5,3 \\
-18,0\end{array}\right|$ & $\left\{\begin{array}{l}69 \text { Std. nach bds. Ureteren- } \\
\text { unterbindung. }\end{array}\right.$ \\
\hline$+34,2$ & $\begin{array}{l}0,414 \\
0,590\end{array}$ & $+42,5$ & $\begin{array}{l}+6,4 \\
+13,0\end{array}$ & $\begin{array}{l}1,49 \\
0,95\end{array}$ & $-36,2$ & $\begin{array}{l}1,38 \\
0,79\end{array}$ & $-42,7$ & $\left|\begin{array}{r}-7,4 \\
-16,8\end{array}\right|$ & \\
\hline
\end{tabular}

Durchtritt des Blutes durch den Muskel geradezu ansteigen, hier ist eher das Gegenteil der Fall. Es ist dies offensichtlich ein Beweiss dafür, dass das Blut beim Durchtritt durch den Muskel an seinen feindispersen Eiweissteilchen darin verliert und grobdisperse Eiweissteilchen aus dem Muskel in sich aufnimmt, d.h. beim Durchtritt des Blutes durch dem Muskel ein besonderer Austausch verschiedener Eiweissteilchen zwischen Blut und Muskelgewebe stattfindet.

Bei der N-Analyse ergibt sich, dass sowohl auf der arteriellen wie auch der venösen Seite Gesamt-N, Rest-N und Globulin-N Zunahmen, Albumin- $\mathrm{N}$ aber hingegen eine Abnahme erfahren, und dass die Grade dieser $\mathrm{Ab}$ - und Zunahmen stets auf der venösen Seite grösser sind. Wenn man obige Grössen im einzelnen dem Durchschnittswert nach gegeneinander vergleicht, so erhält man folgende Zifferzahlen: Gesamt-N, der vor Ureterenunterbindung im Arterienblut einen Wert, $0,992 \mathrm{~g} / \mathrm{dl}$ zeigte, stieg im Venenblut nach dem Durchfliessen des Muskels auf 1,009 g/dl, 
die prozentische Zunahme betrug nur 1,7\%, während Gesamt-N nach Ureterenunterbindung, der sich vor dem Durchfliessen des Muskels auf 1,285 $\mathrm{g} / \mathrm{dl}$ bezifferte, nach demselben auf $1,347 \mathrm{~g} / \mathrm{dl}$ erhöht war, die Zunahme betrug also $4,8 \%$. Rest-N, der vor Ureterenunterbindung vor dem Durchfliessen des Muskels einen Wert von $0,027 \mathrm{~g} / \mathrm{dl}$ aufwies, erhöhte sich nach demselben auf $0,028 \mathrm{~g} / \mathrm{dl}$, die prozentische Zunahme betrug 3,7\%. Nach Ureterenunterbindung stieg Rest-N, der vor dem Durchfliessen des Muskels einen Wert von 0,272 g/dl aufwies, nach demselben auf $0,293 \mathrm{~g} / \mathrm{dl}$ an, die prozentische Zunahme belief sich auf $7,7 \%$, diese Zunahme war beinahe doppelt so gross wie die in der Norm.

Nach obigen Ergebnissen lässt sich also sagen, dass nach Durchtritt des Blutes durch den durch Ureterenunterbindung urämisch geschädigten Muskel Gesamt- $\mathrm{N}$ sowie Rest- $\mathrm{N}$ in erheblichem Masse zunehmen.

Während das Albumin, welches vor Ureterenunterbindung vor dem Durchfliessen des Muskels einen Wert von $0,571 \mathrm{~g} / \mathrm{dl}$ aufwies, nach demselben auf $0,565 \mathrm{~g} / \mathrm{dl}$ abfiel, nämlich eine Abnahme von 1,0\% erfuhr, war das Albumin nach Ureterenunterbindung, welches vor dem Durchfliessen des Muskel einen Wert von $0,490 \mathrm{~g} / \mathrm{dl}$ aufwies, nach demselben auf $0,461 \mathrm{~g} / \mathrm{dl}$ herabgesetzt; die prozentische Abnahme betrug hier 5,9\%. Diese Abnahme ist gegenüber dem Albuminwert nach Durchfliessen des normalen Muskels beträchtlich grösser.

Globulin, welches vor Ureterenunterbindung im Arterienblut einen Wert von $0,389 \mathrm{~g} / \mathrm{dl}$ aufwies, vermehrte sich im Venenblut nach dem Durchfliessen des Muskels auf $0,414 \mathrm{~g} / \mathrm{dl}$, hier erfolgte eine prozentische Zunahme von $6,4 \%$. Nach Ureterenunterbindung vermehrte sich das Globulin, welches im Arterienblut 0,522 g/dl betrug, im Venenblut nach Durchfliessen des Muskels auf $0,590 \mathrm{~g} / \mathrm{dl}$, die prozentische Zunahme belief sich auf 13,0\%. Aus obigen Auseinandersetzungen geht nämlich hervor, dass das Globulin, im Gegensatz zum Albumin, nach Ureterenunterbindung im Blut nach Durchfliessen des Muskels in höherem Masse als in der Norm zunimmt. Das Ergebniss dieser N-Analyse steht in gutem Einklang mit dem Resultat der k.o.D.-Bestimmung. Der Albumin-Globulin-Quotient zeigte mithin im Venenblut nach Ureterenunterbindung den kleinsten Wert.

\section{Zusammenfassung.}

Als bei Hunden, welche der beiderseitigen Nephrektomie oder der Ureterenunterbindung unterworfen waren, urämische Symptome auf- 
traten, wurden an dem M. gastrocnemius zufliessenden arteriellen und dem davon abfliessenden venösen Blut Bestimmungen von Hämoglobin, Eiweiss, k. o.D. sowie auch Gesamt-N, Rest-N, Albumin- und Globulin$\mathrm{N}$ vorgenommen, und die hier gewonnenen Werte wurden mit entsprechenden Werten im gesunden Zustand des Versuchstiers verglichen, was folgendes ergab:

Nach Durchtritt des Blutes durch den urämischen Muskel :

1. Erhöhen sich die Konzentration des Hämoglobins und Eiweisses.

2. Es sinken k. o. D. und Druck pro \% ab.

3. Albumin nimmt ab, Globulin hingegen zu, folglich erfährt der Albumin-Globulin-Quotient eine Erniedrigung.

4. Rest-N nimmt in erheblichem Masse zu.

Oben angeführte Verhältnisse sind, wie bereits in der ersten Mitteilung geschildert wurde; auch beim Muskel des normalen Hundes anzutreffen, die $\mathrm{Ab}$ - und Zunahmen der in Betracht kommenden Werte treten indes beim Muskel des der Nephrektomie oder Ureterenunterbindung unterworfenen Tiers in beträchtlich stärkerem Masse auf.

Vorliegende Versuche wurden nur an einem Muskel angestellt, und da in andern Muskeln des ganzen Körpers gewiss auch gleiche Befunde erhoben werden dürften, so ist anzunehmen, dass beim Bestehen des Nierenfunktionsausfalls, wie es oben erwähnt wurde, die aus den Muskeln des ganzen Körpers abgegebenen Mengen von grobdispersen Eiweisssteilchen und von Rest-N durchaus nicht unerheblich sind. Auch die Albuminmengen, die hierbei in die Muskulatur aufgenommen werden, sind keineswegs gering. Schon lediglich aus der Betrachtung über die Muskulatur allein dürfte es wohl verständlich sein, dass bei intensiven Störungen der Nierenfunktion im Blut das Globulin zu- und das Albumin abnimmt, d. h. die Verschiebung des Bluteiweisses nach grobdisperser Seite hin eintritt, wodurch das Absinken des k.o.D. und die Zunahme des Rest-N verursacht werden. Hiermit glaube ich, in vorliegenden.Versuchen den Nachweis einwandfrei erbracht zu haben, wie wichtige Rolle der Muskel bei qualitativen und quantitativen Veränderungen des Bluteiweisses, die bei Nierenschädigungen auftreten, spielt.

Der Grund für derartige auffallende Veränderungen des Eiweissbildes beim Durchtritt des Blutes durch den Muskel des urämischen Tiers dürfte höchstwahrscheinlich darin zu suchen sein, dass durch den Ausfall der Nierenfunktion giftige Substanzen sich im Blut anhäufen, die zuvörderst auf den Muskel einwirkend, den Eiweissumsatz des Mus- 
kels beeinträchtigen und qualitative und quantitative Veränderungen des Muskeleiweisses hervorrufen, wobei auch zugleich die Veränderung in der Permeabilität der Kapillaren im Muskelinnern eintritt.

\section{Schluss.}

Bei Hunden wurde die beiderseitige Nephrektomie oder die Ureterenunterbindung ausgeführt und nach 2-3 tägigem Ablauf an dem dem M. gastrocnemius zuströmenden arteriellen und dem entströmenden venösen Blut die Eiweisskomponente der beiden Blutarten vergleichend analysiert. Es ergab: Im venösen Blut erhöhen sich die Konzentrationen des Hämoglobins und Serumeiweisses in erheblichem Masse. Das Albumin stürzt ab, das Globulin nimmt hingegen beträchtlich zu, weshalb der Albumin-Globulin-Quotient merklich absinkt. Der kolloid-osmotische Druck sowie Druck pro \% erfahren erhebliche Erniedrigungen. Es tritt eine auffallende Vermehrung des Rest-N auf. Derartige Veränderungen treten nach oben genannten Eingriffen quantitativ in weitgehend stärkerem Masse als bei gesunden Zuständen in Erscheinung. 